\title{
MULTIWAVELENGTH OBSERVATIONS OF THE BE-STAR / X-RAY BINARY EXO2030+375 DURING OUTBURST
}

\author{
A.J. NORTON \\ Dept. of Physics, The Open University, Walton Hall, Milton Keynes MK7 6AA, UK \\ M.J. COE, C. EVERALL and P. ROCHE \\ Astronomy \& Space Physics Group, Univ. of Southampton, Southampton SO9 5NH, UK \\ L. BILDSTEN, D. CHAKRABARTY and T.A. PRINCE \\ Division of Physics, Mathematics \& Astronomy, Caltech, Pasadena, CA 91125, USA \\ M.T. STOLLBERG \\ Department of Physics, University of Alabama, Huntsville, AL 35809, USA \\ and \\ R.B. WILSON \\ Space Sciences Lab., NASA Marshall Space Flight Center, Huntsville, AL 95812, USA
}

\section{The EXO2030+375 System}

EXO2030+375 consists of a neutron star in an eccentric 46 day orbit around a 20th magnitude Be-star companion (Coe et al., 1988; Parmar et al., 1989; Stollberg et al., 1993). The Be-star is thought to be surrounded by a shell/disc of material which is responsible for the infrared excess and Balmer emission lines which are characteristic of Be-stars in general. At periastron, the neutron star passes through this circumstellar material, giving rise to enhanced accretion onto the neutron star surface. As a result of this, the $\mathrm{X}$-ray emission (pulsed at the neutron star spin period of $41.8 \mathrm{~s}$ ) increases dramatically, so producing the transient, outburst behaviour which is commonly seen in Be-star / X-ray binaries.

\section{Observations}

During the periastron passage of June 28th - July 7th 1993, we observed EXO2030+375 using the Palomar 200" telescope, the William Herschel Telescope, the UK Infrared Telescope and the BATSE all sky monitor on the Compton Gamma-Ray Observatory. Hard X-ray measurements with BATSE span the entire period of the outburst, as do the infrared photometric observations, carried out in $\mathrm{J}, \mathrm{H}, \mathrm{K}$ and $\mathrm{L}^{\prime}$ bands. The optical spectroscopic observations were typically at $<1 \AA$ resolution, centred at the wavelengths of $\mathrm{H} \alpha$ and $\mathrm{H} \beta$. These were obtained during the three nights covering the rise of the X-ray outburst. 

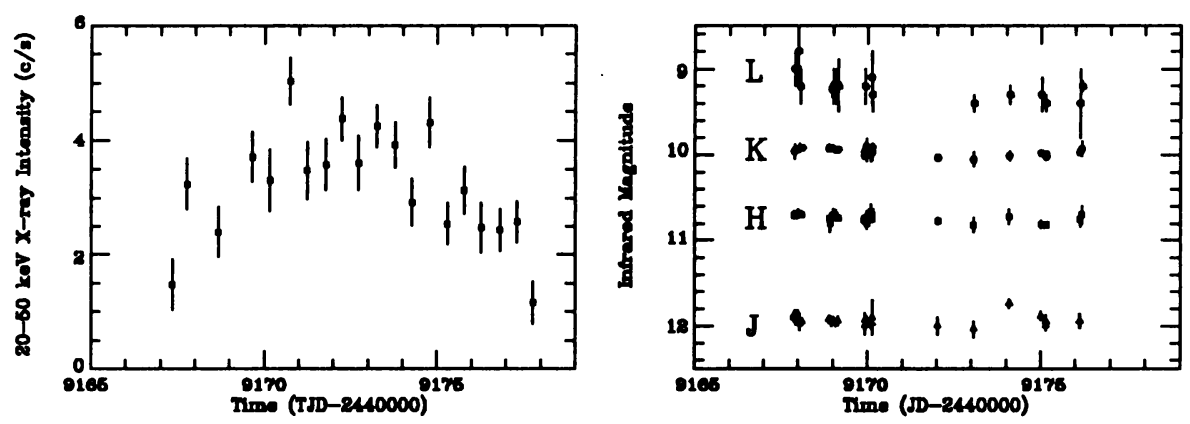

Fig. 1. The X-ray and infrared lightcurves of EXO2030+375 during the outburst

\section{Results}

As can be seen above, the X-ray intensity of EXO2030+375 increased by an order of magnitude over the course of about one week, followed by a decline over a similar timescale. Prior to JD2449167 and after JD2449178, the pulsed $\mathrm{X}$-ray emission from the source was below the limits of detectability. It is clear that the scale of any correlated brightening or fading of the source at infrared wavelengths is less than one-tenth of a magnitude. Similarly the $\mathrm{H} \alpha$ spectroscopy shows no significant changes in either the equivalent width, the intensity or the profile of the $\mathrm{H} \alpha$ emission line. Hence, contrary to intuitive belief, we observed no change in the emission properties of the circumstellar material during this cataclysmic event. That is to say, the passage of the neutron star through the circumstellar material around the Be-star has no detectable effect on the structure or properties of that material.

\section{Why no change ?}

There are two ways in which the passage of the neutron star through the circumstellar material of the Be-star may be expected to influence its emission properties. Firstly, the intense flux of X-rays which is intercepted by the circumstellar material may be reprocessed into optical or infrared emission. Secondly, the passage of the neutron star itself may gravitationally disrupt the structure of the circumstellar disc/shell, so giving rise to changes in its emission characteristics. The fact that no such changes are observed implies that neither of these effects are significant.

\section{References}

Coe, M.J., Longmore, A., Payne, B.J. and Hanson, C.G.: 1988, MNRAS 232, 865

Parmar, A.N., White, N.E., Stella, L., Izzo, C. \& Ferri, P.: 1989, ApJ 338, 359

Stollberg, M.T. et al.: 1993, 'BATSE Observations of EXO2030+375' in Friedlander, Gehrels \& Macomb, ed(s)., CGRO Symposium, St. Louis, AIP Conf. Proc. 280, 371 